\title{
Exceptional Groups and del Pezzo Surfaces
}

\author{
Robert Friedman* and John W. Morgan ${ }^{\dagger}$
}

October 20, 2018

Dedicated to Herb Clemens

\section{Introduction}

Let $\mathbf{E}_{r}, r=6,7,8$ denote the simply connected form of the complex linear group whose root system is of type $E_{r}$. We extend this series to $3 \leq r \leq 8$ by setting $E_{5}=D_{5}, E_{4}=A_{4}$, and $E_{3}=A_{1} \times A_{2}$, again with the understanding that $\mathbf{E}_{r}$ denotes the simply connected form of the corresponding complex linear group of type $E_{r}$. It has long been known that there are deep connections between $\mathbf{E}_{r}$ and del Pezzo surfaces of degree $9-r$. The goal of this paper is to make one of these connections explicit: if $X$ is a del Pezzo surface of degree $d=9-r$, possibly with rational double point singularities, we show that there is a "tautological" holomorphic $\widetilde{\mathbf{E}}_{r}$-bundle $\Xi$ over $X$, where $\widetilde{\mathbf{E}}_{r}$ is an appropriate conformal form of the group $\mathbf{E}_{r}$. The most classical case, $r=6$, corresponds to the case of cubic surfaces. In this case, $\widetilde{\mathbf{E}}_{6}=\mathbf{E}_{6} \times_{\mathbb{Z} / 3 \mathbb{Z}} \mathbb{C}^{*}$. There is a natural 27-dimensional representation $\rho$ of $\mathbf{E}_{6}$ and $\widetilde{\mathbf{E}}_{6}$. If $X$ is a smooth cubic surface, then the induced holomorphic vector bundle $\Xi \times_{\widetilde{\mathbf{E}}_{6}} \mathbb{C}^{27}$ is isomorphic to $\bigoplus_{i=1}^{27} \mathcal{O}_{X}\left(L_{i}\right)$, where the $L_{i}$ are the distinct lines on $X$. The fact that $\Xi \times_{\widetilde{\mathbf{E}}_{6}} \mathbb{C}^{27}$ is isomorphic to a direct sum of line bundles reflects the fact that the structure group of $\Xi$ reduces to a maximal torus of $\widetilde{\mathbf{E}}_{6}$. When $X$ has rational double points, the induced rank 27 vector bundle is no longer a direct sum of line bundles. Instead, the line bundle factors on a general surface coalesce into irreducible summands of higher rank, reflecting the way in which lines coalesce on singular cubic surfaces. Correspondingly, the structure group of $\Xi$ reduces to a reductive subgroup of $\widetilde{\mathbf{E}}_{6}$ whose Lie algebra is generated by a maximal torus and by roots corresponding to smooth rational curves of self-intersection -2 in the minimal resolution of $X$. This phenomenon reflects the picture in physics, where rational double point singularities correspond to extra massless particles, and these particles are described as gauge particles for a gauge group formed in exactly the same way. Similar results hold for any value of $r$.

\footnotetext{
${ }^{*}$ The first author was partially supported by NSF grant DMS-99-70437.

${ }^{\dagger}$ The second author was partially supported by NSF grant DMS-97-04507.
} 
One motivation for describing the bundle $\Xi$ is to give a more direct explanation for the correspondence described in [6] between S-equivalence classes of semistable $\mathbf{E}_{r}$-bundles over a smooth elliptic curve $E$ with origin $p_{0}$ and triples $(X, D, \varphi)$, where $X$ is a del Pezzo surface of degree $9-r, D$ is a hyperplane section of $X$, and $\varphi: D \rightarrow E$ is an isomorphism from $D$ to $E$ such that $\varphi^{*} \mathcal{O}_{E}\left((9-r) p_{0}\right) \cong \mathcal{O}_{X}(D) \mid D$. We show that, given the triple $(X, D, \varphi)$, after a suitable twist by a line bundle, there is a canonical reduction of the structure group of the bundle $\varphi^{*}(\Xi \mid D)$ to an $\mathbf{E}_{r}$-bundle $\xi$ over $E$ which realizes the above correspondence. Moreover, in our construction, the bundle $\xi$ is always the regular representative.

In [8], Manin writes of the 27 lines on a cubic surface, "Their elegant symmetry both enthrals and at the same time irritates; what use is it to know, for instance, the number of coplanar triples of such lines (forty five) or the number of double Schläffli sixfolds (thirty six)?" One answer, different from that given in [8], is that the numerology of the lines, conics, twisted cubics, etc., is deeply related to the weights of the fundamental representations of $\mathbf{E}_{r}$. We give a brief discussion of this in Section 5 .

Much of the material in this paper on del Pezzo surfaces is standard. General references are [8] and [2]. We should also mention that Leung has independently given a different construction of the bundle $\Xi$ in case $X$ is smooth [0].

Let us close this introduction with a list of some outstanding open questions:

1. Is there an algebraic construction of the bundles $\Xi$ along the lines of the parabolic construction of bundles on the hyperplane sections 畉?

2. In the case where $D$ is an irreducible but singular hyperplane section of $X$, passing through a singular point, and $\varphi$ is an isomorphism from $D$ to a fixed Weierstrass cubic $E$, can one relate the bundle $\xi$ constructed above to the $\mathbf{E}_{r}$-bundle constructed in [5]?

3. Can the construction be extended to certain more singular del Pezzo surfaces, in particular the non-normal del Pezzo surfaces of Reid [9]? What about weighted cones over elliptic curves?

4. Is there a general method of constructing $\Xi$ for families of del Pezzo surfaces $\pi: \mathcal{X} \rightarrow B$ over a base $B$ ? Does some variant of the relative intermediate Jacobian of $\mathcal{X}$ play a role in describing all such bundles?

\section{Some results on lattices}

Let $\Lambda$ be a lattice. In practice, $\Lambda$ will be the coroot lattice of the simply connected group $G$, or $\Lambda$ will be $\left[K_{\tilde{X}}\right]^{\perp} \subseteq H^{2}(\widetilde{X} ; \mathbb{Z})$, where $\widetilde{X}$ is the minimal resolution of a del Pezzo surface with rational double points. We consider extensions

$$
0 \rightarrow \mathbb{Z} \oplus \Lambda \rightarrow \widetilde{\Lambda} \rightarrow \mathbb{Z} / d \mathbb{Z} \rightarrow 0
$$


where $\widetilde{\Lambda}$ is also a lattice. Let $\kappa$ be the image of $(1,0)$, and identify $\Lambda$ with its image. We assume that $\Lambda$ is a primitive sublattice of $\widetilde{\Lambda}$, and that $\kappa$ is a primitive vector. There are then two related exact sequences

$$
\begin{aligned}
& 0 \rightarrow \Lambda \rightarrow \widetilde{\Lambda} \rightarrow \mathbb{Z} \rightarrow 0 \\
& 0 \rightarrow \mathbb{Z} \rightarrow \widetilde{\Lambda} \rightarrow \bar{\Lambda} \rightarrow 0 .
\end{aligned}
$$

Choose a generator of $\mathbb{Z} / d \mathbb{Z}$, and lift it to an element $\mu \in \widetilde{\Lambda}$. We may write $\mu=\mu_{1}+\mu_{2}$, where $\mu_{1} \in \frac{1}{d} \mathbb{Z}$ and $\mu_{2} \in \frac{1}{d} \Lambda$. It is easy to check that our hypotheses imply that $\mu_{1}$ has order $d$ as an element of $\frac{1}{d} \mathbb{Z} / \mathbb{Z}$, and similarly for $\mu_{2}$. We can choose the generator of $\mathbb{Z} / d \mathbb{Z}$ uniquely so that $\mu_{1} \equiv 1 / d \bmod \mathbb{Z}$, and then $\mu_{2}$ is a well-defined element of $\frac{1}{d} \Lambda / \Lambda$ and is a complete invariant of the extension.

Suppose that there is a unimodular quadratic form on $\widetilde{\Lambda}$ such that $\kappa$ and $\Lambda$ are orthogonal. In this case, $\mu_{2}$ is a well-defined element of $\Lambda^{*} / \Lambda$. Given $\lambda \in \widetilde{\Lambda}$, we can write $\lambda=\lambda_{1}+\lambda_{2}$, where the $\lambda_{i} \in \widetilde{\Lambda} \otimes \mathbb{Q}, \lambda_{1}=\langle\lambda, \kappa\rangle \kappa / \kappa^{2}$, and $\lambda_{2} \in \kappa^{\perp}$. Thus $\lambda_{i} \in \widetilde{\Lambda}$ if and only if $\kappa^{2} \mid\langle\lambda, \kappa\rangle$, and the index $d$ of $\mathbb{Z} \oplus \Lambda$ in $\widetilde{\Lambda}$ is $\left|\kappa^{2}\right|$. We can choose a representative for $\mu_{2}$ as follows: first choose $\mu \in \widetilde{\Lambda}$ such that $\langle\mu, \kappa\rangle=1$. Then we can take

$$
\mu_{2} \equiv \mu-\frac{1}{\kappa^{2}} \kappa \quad \bmod \Lambda \text {. }
$$

In fact, $\Lambda^{*} / \Lambda$ is cyclic of order $d$ and $\mu_{2}$ is a generator.

Example: Suppose that $3 \leq r \leq 8$. If $\widetilde{\Lambda}=\mathbb{Z}^{r+1}$ with diagonal basis $h, e_{1}, \ldots, e_{r}$, where $h^{2}=1$ and $e_{i}^{2}=-1$, let $\kappa=3 h-\sum_{i} e_{i}$ and $\Lambda=\kappa^{\perp}$. Take $\mu=e_{r}$. A basis for $\Lambda$ is given by $\alpha_{1}=e_{1}-e_{2}, \ldots, \alpha_{r-1}=e_{r-1}-e_{r}, \alpha_{r}=3 h-e_{1}-e_{2}-e_{3}$. In this case $\mu_{2}=\varpi_{r-1}$ is the dual basis element to $\alpha_{r-1}$.

The lattice $\Lambda$ is the coroot lattice for $\mathbf{E}_{r}$, with the negative of the usual intersection form. The action of the Weyl group $W$ generated by reflections in the roots extends to an action of $W$ on $\widetilde{\Lambda}$. We have the following:

Lemma 1.1. The group $W$ is exactly the subgroup of automorphisms of $\widetilde{\Lambda}$ which fix $\kappa$.

Proof. The automorphism group of $\Lambda$ is a semidirect product of $W$ and the outer automorphism group of the root system corresponding to $\Lambda$. It is easy to verify that, in the cases at hand, a nontrivial outer automorphism acts by sending $\mu_{2}$ to $-\mu_{2} \bmod \Lambda$. From this, the result is clear.

The intersection form identifies $\widetilde{\Lambda}$ with its dual. Using this identification, we have exact sequences

$$
\begin{gathered}
0 \rightarrow \mathbb{Z} \rightarrow \widetilde{\Lambda} \rightarrow \Lambda^{*} \rightarrow 0 ; \\
0 \rightarrow \widetilde{\Lambda} \rightarrow(\mathbb{Z} \oplus \Lambda)^{*} \rightarrow \mathbb{Z} / d \mathbb{Z} \rightarrow 0 .
\end{gathered}
$$

From these sequences, we see the following: 
Lemma 1.2. (i) A homomorphism $\phi: \mathbb{Z} \oplus \Lambda \rightarrow \mathbb{Z}$ is given by inner product with an element of $\widetilde{\Lambda}$ if and only if $\phi(1,0) \equiv-\phi\left(0, d \mu_{2}\right) \bmod d$.

(ii) Given $\phi \in \Lambda^{*}$, there exists a $\lambda \in \Lambda$ such that $\phi(v)=\langle\lambda$, $v\rangle$ for all $v \in \Lambda$. Moreover, any two choices of $\lambda$ differ by an integral multiple of $\kappa$.

\section{A tautological bundle over the Cartan subgroup of a con- formal form}

We begin by constructing the appropriate conformal form of the group $\mathbf{E}_{r}$. More generally, suppose that $G$ is a reductive group with Cartan subgroup $H$, and that $c$ is an element of the center of $G$ of finite order $d$. Let $\Lambda=\pi_{1}(H)$. We can then form the group $\widetilde{G}=\mathbb{C}^{*} \times_{\mathbb{Z} / d \mathbb{Z}} G$, where the generator $1 \in \mathbb{Z} / d \mathbb{Z}$ maps to $\exp (2 \pi \sqrt{-1} / d) \in \mathbb{C}^{*}$ and to $c \in G$. A Cartan subgroup of $\widetilde{G}$ is given by $\widetilde{H}=\mathbb{C}^{*} \times_{\mathbb{Z} / d \mathbb{Z}} H$. Let $\widetilde{\Lambda}=\pi_{1}(\widetilde{H})$. From the exact sequence

$$
0 \rightarrow \mathbb{Z} / d \mathbb{Z} \rightarrow \mathbb{C}^{*} \times H \rightarrow \mathbb{C}^{*} \times_{\mathbb{Z} / d \mathbb{Z}} H \rightarrow 0,
$$

we see that there is an exact sequence

$$
0 \rightarrow \mathbb{Z} \oplus \Lambda \rightarrow \widetilde{\Lambda} \rightarrow \mathbb{Z} / d \mathbb{Z} \rightarrow 0
$$

and that we obtain the extension of tori by tensoring the above sequence with $\mathbb{C}^{*}$. It is an easy exercise to check that the element $\mu_{2}$ defined in the previous section is just the element $c^{-1}$, viewed as an element of $\Lambda^{*} / \Lambda \subseteq \Lambda \otimes \mathbb{Q} / \Lambda$. Conversely, given an extension $\widetilde{\Lambda}$ of $\mathbb{Z} \oplus \Lambda$ with a unimodular form as in the previous section, suppose that the element $\mu_{2}$ corresponds to the element $c^{-1} \in \Lambda^{*} / \Lambda$. If we tensor with $\mathbb{C}^{*}$, we get an extension of tori and the torus $\widetilde{H}=\widetilde{\Lambda} \otimes_{\mathbb{Z}} \mathbb{C}^{*}$ is a Cartan subgroup of $\widetilde{G}$.

We apply this to the lattice $\widetilde{\Lambda}=\mathbb{Z}^{r+1}$ with basis $h, e_{1}, \ldots, e_{r}$ of the example given at the end of the previous section. In this case, $\Lambda$ is identified with the coroot lattice of the group $\mathbf{E}_{r}$ (with the negative of the usual intersection form), and thus $\widetilde{H}=\widetilde{\Lambda} \otimes_{\mathbb{Z}} \mathbb{C}^{*}$ is a Cartan subgroup for $\widetilde{\mathbf{E}}_{r}=\mathbf{E}_{r} \times_{\mathbb{Z} /(9-r) \mathbb{Z}} \mathbb{C}^{*}$. Note that, for $3 \leq r \leq 8$ and $r \neq 4$, the group $\mathbb{Z} /(9-r) \mathbb{Z}$ has at most two generators and there is essentially no choice for the group $\widetilde{\mathbf{E}}_{r}$. However, for $r=4$, the central element $c$ is the square of the standard generator of the center of $S L_{5}(\mathbb{C})$, and $\widetilde{\mathbf{E}}_{4}$ is not $G L_{5}(\mathbb{C})$ but rather the double cover of $G L_{5}(\mathbb{C})$.

Now let $X$ be a del Pezzo surface of degree $9-r$ with at worst rational double point singularities and let $\widetilde{X}$ be the minimal resolution of $X$. Fix an isomorphism $\psi: H^{2}(\widetilde{X} ; \mathbb{Z}) \rightarrow$ $\widetilde{\Lambda}$ such that $\psi\left(\left[K_{X}\right]\right)=-\kappa$. We will refer to such a $\psi$ as a marking. It follows from Lemma 1.1 that any two markings differ by an action of the Weyl group on $\widetilde{\Lambda}$.

Holomorphic $\widetilde{H}$-bundles over $\widetilde{X}$ are classified by $H^{1}(\widetilde{\widetilde{X}} ; \underline{\widetilde{H}})$, where $\underline{\widetilde{H}}$ is the sheaf whose sections over an open set $U$ are holomorphic maps from $U$ to $\widetilde{H}$. From the exact sequence

$$
0 \rightarrow \widetilde{\Lambda} \rightarrow \operatorname{Lie}(\widetilde{H}) \otimes \mathcal{O}_{\widetilde{X}} \rightarrow \underline{\widetilde{H}} \rightarrow\{1\},
$$


and the fact that $H^{i}\left(\widetilde{X} ; \mathcal{O}_{\widetilde{X}}\right)=0$ for $i>0$, we see that there are canonical isomorphisms

$$
H^{1}(\widetilde{\widetilde{X}} ; \underline{\widetilde{H}}) \cong H^{2}(\widetilde{X} ; \widetilde{\Lambda}) \cong H^{2}(\widetilde{X} ; \mathbb{Z}) \otimes_{\mathbb{Z}} \widetilde{\Lambda} .
$$

The marking $\psi$ defines an isomorphism $H^{2}(\widetilde{X} ; \mathbb{Z}) \otimes_{\mathbb{Z}} \widetilde{\Lambda} \cong \widetilde{\Lambda} \otimes_{\mathbb{Z}} \widetilde{\Lambda}$. Using the intersection form to identify $\widetilde{\Lambda}$ with its dual, we can take the class of $\operatorname{Id} \in \widetilde{\Lambda}^{*} \otimes_{\mathbb{Z}} \widetilde{\Lambda} \cong \widetilde{\Lambda} \otimes_{\mathbb{Z}} \widetilde{\Lambda}$. Let $\Xi_{\widetilde{H}}$ be the corresponding $\widetilde{H}$-bundle over $\widetilde{X}$, and define

$$
\Xi_{\text {split }}=\Xi_{\widetilde{H}} \times_{\widetilde{H}} \widetilde{\mathbf{E}}_{r} .
$$

The bundle $\Xi_{\text {split }}$ is split, in the sense that, given any representation $\rho: \widetilde{\mathbf{E}}_{r} \rightarrow G L(N)$, the induced vector bundle $\Xi_{\text {split }} \times_{\widetilde{\mathbf{E}}_{r}} \mathbb{C}^{N}$ is a direct sum of line bundles. Since any two markings differ by an element of the Weyl group, it is clear that the isomorphism class of the $\widetilde{\mathbf{E}}_{r}$-bundle $\Xi_{\text {split }}$ is independent of the choice of a marking. In case $\widetilde{X}=X$, i.e. in the case of a smooth del Pezzo surface, we take $\Xi=\Xi_{\text {split }}$. In the next section, we will discuss how to modify $\Xi_{\text {split }}$ so as to make it trivial in a neighborhood of the preimages of the double points on $X$.

Let us record one basic property of $\Xi_{\text {split }}$. Suppose that $\lambda \in H^{2}(\widetilde{X} ; \mathbb{Z})$. Using the marking to view $\psi$ as an element of $\widetilde{\Lambda}$ and the quadratic form, we can view $\lambda$ as a homomorphism $\widetilde{\Lambda} \rightarrow \mathbb{Z}$. Hence $\lambda$ defines a homomorphism $\widetilde{H} \rightarrow \mathbb{C}^{*}$. There is the associated $\mathbb{C}^{*}$-bundle $\Xi_{\text {split }} \times \widetilde{H} \mathbb{C}^{*}$, which corresponds to a complex line bundle $L_{\lambda}$ on $\widetilde{X}$. Unwinding the definitions gives:

Lemma 2.1. In the above notation, $c_{1}\left(L_{\lambda}\right)=\lambda$.

\section{Extension over the Borel subgroup}

The bundle $\Xi_{\text {split }}$ is the correct bundle only when $X=\tilde{X}$, i.e. $X$ is smooth. In case $X$ is singular, there are rational curves $C$ on $\widetilde{X}$ of self-intersection -2 , which for brevity we call -2-curves. Define the exceptional divisor $Y$ on $\widetilde{X}$ to be the union of the -2 -curves. The bundle $\Xi_{\text {split }}$ is nontrivial in every neighborhood of a -2-curve, and hence does not descend to a bundle on $X$. In this section, we show how to modify the bundle $\Xi_{\text {split }}$ so that it becomes trivial in a neighborhood of the exceptional divisor. The idea is as follows. Fix a general ample line bundle $L$ on $\widetilde{X}$. Then $\psi(L)$ defines a positive Weyl chamber in $\Lambda$ and hence a Borel subgroup of $\mathbf{E}_{r}$ and thus of $\widetilde{\mathbf{E}}_{r}$. Let $U$ be the unipotent radical of this Borel subgroup, so that the Borel is equal to $\widetilde{H} U$. We will construct a lift of the $\widetilde{H}$-bundle $\Xi_{\widetilde{H}}$ to an $\widetilde{H} U$-bundle $\Xi_{\widetilde{H} U}$, so that $\Xi_{\widetilde{H} U} \times \widetilde{H} U \widetilde{\mathbf{E}}_{r}$ is trivial in an analytic neighborhood of the exceptional divisor, and hence arises from a bundle over $X$. The results are summarized by the following theorem:

Theorem 3.1. There exists a unique $\widetilde{\mathbf{E}}_{r}$-bundle $\widetilde{\Xi}$ over $\widetilde{X}$ with the following properties:

(i) There exists a bundle $\Xi_{\widetilde{H} U}$, where $U$ is the unipotent radical of the Borel subgroup of $\widetilde{\mathbf{E}}_{r}$, such that $\Xi_{\widetilde{H} U} / U=\Xi_{\text {split }}$ and $\widetilde{\Xi}=\Xi_{\widetilde{H} U} \times \widetilde{H} U_{r} \widetilde{\mathbf{E}}_{r}$. 
(ii) The bundle $\widetilde{\Xi}$ is rigid, i.e. $H^{1}(\widetilde{X} ;$ ad $\widetilde{\Xi})=0$.

In case $X=\widetilde{X}, \widetilde{\Xi}=\Xi_{\text {split }}$. Furthermore, the bundle $\widetilde{\Xi}$ has the following properties:

(iii) For every $D \in\left|-K_{\widetilde{X}}\right|, h^{0}(D ; \operatorname{ad} \widetilde{\Xi} \mid D)=r+1$.

(iv) There exists a unique $\widetilde{\mathbf{E}}_{r}$-bundle $\Xi$ on $X$ such that $\widetilde{\Xi}=p^{*} \Xi$.

The proof will be in several steps. We begin with some very general and well-known results about -2 -curves on the minimal resolution of a del Pezzo surface $X$ with rational double points. Let $p: \widetilde{X} \rightarrow X$ be the minimal resolution of $X$. Given $\alpha \in H^{2}(\widetilde{X} ; \mathbb{Z})$, we call $\alpha$ a root if $\alpha^{2}=-2$ and $\alpha \cdot K_{\tilde{X}}=0$. For any class $\alpha \in H^{2}(\widetilde{X} ; \mathbb{Z})$, let $L_{\alpha}$ be the corresponding line bundle.

Lemma 3.2. Let $\alpha$ be a root, let $C_{1}, \ldots, C_{k}$ be -2 curves in $\widetilde{X}$, and let $a_{i} \in \mathbb{Z}$. Then

$$
H^{2}\left(\widetilde{X} ; L_{\alpha} \otimes \mathcal{O}_{\tilde{X}}\left(\sum_{i} a_{i} C_{i}\right)\right)=0 .
$$

Moreover, the following are equivalent:

(i) $H^{1}\left(\widetilde{X} ; L_{\alpha}\right) \neq 0$;

(ii) $H^{1}\left(\widetilde{X} ; L_{\alpha}\right) \cong \mathbb{C}$;

(iii) $H^{0}\left(\widetilde{X} ; L_{\alpha}\right) \neq 0$, i.e. $L_{\alpha}=\mathcal{O}_{\widetilde{X}}(C)$ for some effective curve $C$;

(iv) $H^{0}\left(\widetilde{X} ; L_{\alpha}\right) \cong \mathbb{C}$.

Proof. By Serre duality, $H^{2}\left(\widetilde{X} ; L_{\alpha}\right) \cong H^{0}\left(\widetilde{X} ; K_{\widetilde{X}} \otimes L_{\alpha}^{-1}\right)$. Since $K_{\widetilde{X}}=\mathcal{O}_{\widetilde{X}}(-D)$, where $D$ is nef and big, and $D \cdot \alpha=0, H^{2}\left(\widetilde{X} ; L_{\alpha}\right)=0$ for every root $\alpha$. A similar statement holds for $H^{2}\left(\widetilde{X} ; L_{\alpha} \otimes \mathcal{O}_{\widetilde{X}}\left(\sum_{i} a_{i} C_{i}\right)\right)$.

Let $\alpha$ be a root. Then $\alpha^{2}=-2$, and so by the Riemann-Roch theorem, $\chi\left(\widetilde{X} ; L_{\alpha}\right)=0$. By the first part of the lemma, $h^{2}\left(\widetilde{X} ; L_{\alpha}\right)=0$ and so $h^{1}\left(\widetilde{X} ; L_{\alpha}\right)=h^{0}\left(\widetilde{X} ; L_{\alpha}\right)$. Thus $h^{1}\left(\widetilde{X} ; L_{\alpha}\right) \neq 0$ if and only if $L_{\alpha}=\mathcal{O}_{\widetilde{X}}(C)$ for some effective curve $C$. In this case, since $D \cdot C=0$ and $D$ is nef and big, it follows that $h^{0}\left(\widetilde{X} ; L_{\alpha}\right)=1$, and hence that $h^{1}\left(\widetilde{X} ; L_{\alpha}\right)=1$. The remaining equivalences are clear.

If $\alpha$ satisfies any of the equivalent conditions above, we call $\alpha$ an effective root.

Next, we see how the line bundles $L_{\alpha}$ restrict to smooth elements $D \in\left|-K_{\tilde{X}}\right|$ :

Lemma 3.3. Let $\alpha$ be a root. Suppose that $D$ is reduced and irreducible and that $K_{\widetilde{X}}=$ $\mathcal{O}_{\widetilde{X}}(-D)$. Then the following are equivalent:

(i) $L_{\alpha} \mid D=\mathcal{O}_{D}$;

(ii) Either $\alpha$ or $-\alpha$ is effective. 
Moreover, in this case $\alpha$ is effective if and only if $L \cdot \alpha>0$. Finally, for every root $\alpha$, if $L \cdot \alpha>0$, then the natural maps $H^{i}\left(\widetilde{X} ; L_{\alpha}\right) \rightarrow H^{i}\left(D ; L_{\alpha} \mid D\right)$ are all isomorphisms.

Proof. In any case $L_{\alpha} \mid D$ has degree zero. Suppose that $-\alpha$ is not effective. Then $H^{i}\left(\widetilde{X} ; L_{\alpha}^{-1}\right)=0$ for all $i$. Consider the long exact cohomology sequence arising from

$$
0 \rightarrow \mathcal{O}_{\tilde{X}}(-D) \otimes L_{\alpha} \rightarrow L_{\alpha} \rightarrow L_{\alpha} \mid D \rightarrow 0 .
$$

Since $H^{i}\left(\widetilde{X} ; \mathcal{O}_{\widetilde{X}}(-D) \otimes L_{\alpha}\right)$ is Serre dual to $H^{2-i}\left(\widetilde{X} ; L_{\alpha}^{-1}\right)$, it is zero for all $i$. Thus $H^{i}\left(\tilde{X} ; L_{\alpha}\right) \rightarrow H^{i}\left(D ; L_{\alpha} \mid D\right)$ is an isomorphism for all $i$. It follows that $\alpha$ is effective if and only if $H^{0}\left(D ; L_{\alpha} \mid D\right) \neq 0$, if and only if $L_{\alpha} \mid D$ is trivial (since it is a line bundle of degree zero). The remaining statements are clear.

The next lemma gives a necessary and sufficient condition for the bundle $\widetilde{\Xi}$ to descend to a bundle on the singular surface $X$.

Lemma 3.4. Let $\widetilde{\Xi}$ be an $\widetilde{\mathbf{E}}_{r}$-bundle over $\widetilde{X}$. Suppose that $\widetilde{\Xi} \mid C$ is the trivial $\widetilde{\mathbf{E}}_{r}$-bundle for every irreducible -2-curve $C$. Then there exists a unique $\widetilde{\mathbf{E}}_{r}$-bundle $\Xi$ on $X$ such that $\widetilde{\Xi}=p^{*} \Xi$.

Proof. Let $V$ be a vector bundle on $\widetilde{X}$ such that $V \mid C \cong \mathcal{O}_{C}^{n}$ for every irreducible -2-curve $C$. We shall show that $V$ is trivial in some analytic neighborhood of the exceptional curve $Y$. If $C_{1}, \ldots, C_{e}$ are the -2-curves, and $Y=\bigcup_{i} C_{i}$, it follows easily from the fact that the dual graph of $Y$ is a union of contractible components and that the $C_{i}$ meet transversally that

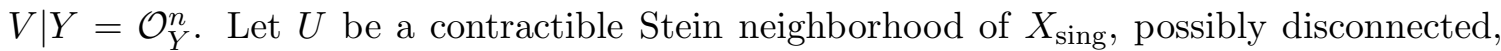
and let $\widetilde{U}=p^{-1}(U)$. We claim that the natural map $H^{0}(\widetilde{U} ; V \mid \widetilde{U}) \rightarrow H^{0}(Y ; V \mid Y)$ is surjective. In fact, the cokernel of this map is contained in $H^{1}\left(\widetilde{U} ; V \otimes \mathcal{O}_{\widetilde{X}}(-Y) \mid \widetilde{U}\right) \cong$ $H^{0}\left(R^{1} p_{*}\left(V \otimes \mathcal{O}_{\widetilde{X}}(-Y)\right)\right)$. A standard argument using the fact that the singularities are rational double points and the formal functions theorem shows that $R^{1} p_{*}\left(V \otimes \mathcal{O}_{\tilde{X}}(-Y)\right)=0$. Thus $H^{0}(\widetilde{U} ; V \mid \widetilde{U}) \rightarrow H^{0}(Y ; V \mid Y)$ is surjective. Lifting a basis of sections of $V \mid Y$ to $\widetilde{U}$, we can assume, possibly after shrinking $U$ and $\widetilde{U}$, that $V \mid \widetilde{U}$ is trivial.

Now choose a faithful representation $\rho$ of $\widetilde{\mathbf{E}}_{r}$, which we use to view $\widetilde{\mathbf{E}}_{r}$ as a subgroup of $G L(N)$ for some $N$. Let $V$ be the induced vector bundle $\widetilde{\Xi} \times_{\widetilde{\mathbf{E}}_{r}} \mathbb{C}^{N}$. By assumption, $V \mid C$ is the trivial vector bundle $\mathcal{O}_{C}^{N}$ for every -2-curve $C$. Thus, $V \mid \widetilde{U}$ is the trivial vector bundle over $\widetilde{U}$.

Let $Q$ be the quotient space $G L(N) / \widetilde{\mathbf{E}}_{r}$, which exists as an affine variety (since $\widetilde{\mathbf{E}}_{r}$ is reductive) and hence as a complex manifold. Let $\underline{Q}$ be the sheaf of morphisms from $\widetilde{U}$ to $Q$, and similarly for $\underline{\widetilde{\mathbf{E}}_{r}}$ and $\underline{G L(N)}$. From the exact sequence of pointed sets

$$
\{1\} \rightarrow \underline{\widetilde{\mathbf{E}}_{r}} \rightarrow \underline{G L(N)} \rightarrow \underline{Q} \rightarrow\{1\}
$$

we have a long exact cohomology sequence through the term $H^{1}(\widetilde{U} ; G L(N))$. If $\bar{\xi}$ is the class in $H^{1}\left(\widetilde{U} ; \underline{\widetilde{\mathbf{E}}_{r}}\right)$ induced by $\widetilde{\Xi} \mid \widetilde{U}$, then $\bar{\xi}$ maps to the trivial element in $H^{1}(\widetilde{U} ; \underline{G L(N))}$, 
and hence comes by coboundary from $H^{0}(\widetilde{U} ; \underline{Q})$, which we can identify with the set of morphisms from $\widetilde{U}$ to $Q$. If $f$ is such a morphism, then since $Q$ is affine, $f(Y)=$ pt. Since $U$ is normal, it follows that $f$ is induced from a morphism $U \rightarrow Q$. But since there are local cross sections for the map $G L(N) \rightarrow Q$ (in the classical topology), again after shrinking $U$ we can assume that $f$ is in the image of a morphism from $\widetilde{U}$ to $G L(N)$. In particular, this says that $\bar{\xi}$ is trivial. Thus the triviality of $V \mid \widetilde{U}$ implies that of $\widetilde{\Xi} \mid \widetilde{U}$. Choosing a local trivialization of $\widetilde{\Xi}$ for which $\widetilde{U}$ is one of the open sets and the remaining ones do not meet $Y$ gives a 1-cocycle defining $\widetilde{\Xi}$ which also defines a bundle $\Xi$ on $X$, and clearly $\widetilde{\Xi}=p^{*} \Xi$. The uniqueness is a straightforward consequence of the fact that, as $\widetilde{\mathbf{E}}_{r}$ is affine, every morphism from $\widetilde{U}$ into $\widetilde{\mathbf{E}}_{r}$ is constant on the exceptional curves and hence descends to a morphism from $U$ into $\widetilde{\mathbf{E}}_{r}$.

We turn now to the construction of a suitable bundle $\widetilde{\Xi}$, which we shall always take to be of the form $\Xi_{\widetilde{H} U} \times \widetilde{H} U_{r} \widetilde{\mathbf{E}}_{r}$, where $\Xi_{\widetilde{H} U}$ is a lift of the $\widetilde{H}$-bundle $\Xi_{\widetilde{H}}$ to an $\widetilde{H} U$-bundle.

Lemma 3.5. Let $\widetilde{\Xi}=\Xi_{\widetilde{H} U} \times_{\widetilde{H} U} \widetilde{\mathbf{E}}_{r}$, where $\Xi_{\widetilde{H} U}$ is a lift of the $\widetilde{H}$-bundle $\Xi_{\widetilde{H}}$ to an $\widetilde{H} U$ bundle. Then the following are equivalent:

(i) $H^{1}(\widetilde{X} ; \operatorname{ad} \widetilde{\Xi})=0$;

(ii) $h^{0}(\widetilde{X} ; \operatorname{ad} \widetilde{\Xi})=r+1$;

(iii) There exists a smooth $D \in\left|-K_{\widetilde{X}}\right|$ such that $h^{0}(D ; \operatorname{ad} \widetilde{\Xi} \mid D)=r+1$;

(iv) For every $D \in\left|-K_{\widetilde{X}}\right|, h^{0}(D ; \operatorname{ad} \widetilde{\Xi} \mid D)=r+1$.

Proof. First we claim:

Lemma 3.6. With $\widetilde{\Xi}$ as in the statement of Lemma 3.5,

(i) $\chi(\widetilde{X} ; \operatorname{ad} \widetilde{\Xi})=r+1$;

(ii) Let $C_{1}, \ldots, C_{k}$ be-2-curves and let $a_{i} \in \mathbb{Z}$. Then

$$
H^{2}\left(\widetilde{X} ; \operatorname{ad} \widetilde{\Xi} \otimes \mathcal{O}_{\widetilde{X}}\left(\sum_{i} a_{i} C_{i}\right)\right)=0=H^{0}\left(\widetilde{X} ; \operatorname{ad} \widetilde{\Xi} \otimes \mathcal{O}_{\widetilde{X}}\left(\sum_{i} a_{i} C_{i}\right) \otimes \mathcal{O}_{\widetilde{X}}(-D)\right)=0 .
$$

Proof. (i) By Riemann-Roch, $\chi(\widetilde{X}$; ad $\widetilde{\Xi})$ only depends on the topological type of $\widetilde{\Xi}$. Thus we may replace $\widetilde{\Xi}$ by $\Xi_{\text {split }}$. But

$$
\operatorname{ad} \Xi_{\text {split }}=\mathcal{O}_{\widetilde{X}}^{r+1} \oplus \bigoplus_{\alpha \in R} L_{\alpha}
$$

and as we have seen, $\chi\left(\widetilde{X} ; L_{\alpha}\right)=0$ for every $\operatorname{root} \alpha$. Hence $\chi(\widetilde{X} ; \operatorname{ad} \widetilde{\Xi})=(r+1) \chi\left(\widetilde{X} ; \mathcal{O}_{\widetilde{X}}\right)=$ $r+1$.

(ii) The bundle ad $\widetilde{\Xi}$ has a filtration whose successive quotients are either $\mathcal{O}_{\widetilde{X}}$ or $L_{\alpha}$, $\alpha \in R$. Thus, by Lemma 3.2, $H^{2}\left(\widetilde{X}\right.$; ad $\left.\widetilde{\Xi} \otimes \mathcal{O}_{\widetilde{X}}\left(\sum_{i} a_{i} C_{i}\right)\right)=0$, and a similar argument shows that $H^{0}\left(\widetilde{X} ; \operatorname{ad} \widetilde{\Xi} \otimes \mathcal{O}_{\widetilde{X}}\left(\sum_{i} a_{i} C_{i}\right) \otimes \mathcal{O}_{\widetilde{X}}(-D)\right)=0$. 
Returning to the proof of Lemma 3.5, we see that

$$
h^{0}(\widetilde{X} ; \operatorname{ad} \widetilde{\Xi})-h^{1}(\widetilde{X} ; \operatorname{ad} \widetilde{\Xi})=\chi(\widetilde{X} ; \operatorname{ad} \widetilde{\Xi})=r+1 .
$$

Thus $h^{0}(\widetilde{X}$; ad $\widetilde{\Xi}) \geq r+1$, with equality if and only if $h^{1}(\widetilde{X}$; ad $\widetilde{\Xi})=0$. In particular, (i) $\Longleftrightarrow$ (ii). Let $D \in\left|-K_{\tilde{X}}\right|$. From the exact sequence

$$
0 \rightarrow \operatorname{ad} \widetilde{\Xi} \otimes \mathcal{O}_{\widetilde{X}}(-D) \rightarrow \operatorname{ad} \widetilde{\Xi} \rightarrow \operatorname{ad} \widetilde{\Xi} \mid D \rightarrow 0,
$$

and the fact that $H^{1}\left(\widetilde{X} ; \operatorname{ad} \widetilde{\Xi} \otimes \mathcal{O}_{\widetilde{X}}(-D)\right)$ is Serre dual to $H^{1}(\widetilde{X}$; ad $\widetilde{\Xi})$, it follows that, if $h^{1}(\widetilde{X} ; \operatorname{ad} \widetilde{\Xi})=0$, then $h^{0}(D ; \operatorname{ad} \widetilde{\Xi} \mid D)=h^{0}(\widetilde{X} ; \operatorname{ad} \widetilde{\Xi})=r+1$. Thus $(\mathrm{i}) \Longrightarrow$ (iv) $\Longrightarrow$ (iii). Finally, since $H^{0}\left(\widetilde{X} ; \operatorname{ad} \widetilde{\Xi} \otimes \mathcal{O}_{\widetilde{X}}(-D)\right)=0$, the map $H^{0}(\widetilde{X} ; \operatorname{ad} \widetilde{\Xi}) \rightarrow H^{0}(D ; \operatorname{ad} \widetilde{\Xi} \mid D)$ is injective. As we have seen, $h^{0}(\widetilde{X} ; \operatorname{ad} \widetilde{\Xi}) \geq r+1$. Thus, if $h^{0}(D ; \operatorname{ad} \widetilde{\Xi} \mid D)=r+1$, then $h^{0}(\tilde{X} ; \operatorname{ad} \widetilde{\Xi})=r+1$ as well, so that (iii) $\Longrightarrow$ (ii).

Now let us analyze the restriction of a bundle $\widetilde{\Xi}$ satisfying any of the equivalent hypotheses of Lemma 3.5 to a -2-curve.

Lemma 3.7. Let $\widetilde{\Xi}=\Xi_{\widetilde{H} U} \times{ }_{\widetilde{H} U} \widetilde{\mathbf{E}}_{r}$, where $\Xi_{\widetilde{H} U}$ is a lift of the $\widetilde{H}$-bundle $\Xi_{\widetilde{H}}$ to an $\widetilde{H} U$ bundle. Suppose that $H^{1}(\widetilde{X} ; \operatorname{ad} \widetilde{\Xi})=0$. Then, for every-2-curve $C, \widetilde{\Xi} \mid C$ is the trivial $\widetilde{\mathbf{E}}_{r}$-bundle.

Proof. First, we claim that $\widetilde{\Xi} \mid C$ is topologically trivial. It suffices to check that $\Xi_{\text {split }} \mid C$ is topologically trivial. The surjection $\widetilde{\mathbf{E}}_{r} \rightarrow \mathbb{C}^{*}$ induces a $\mathbb{C}^{*}$-bundle $\operatorname{det}\left(\Xi_{\text {split }} \mid C\right)$, and the corresponding line bundle is easily seen to be $K_{\tilde{X}} \mid C$, which is trivial. Hence $\Xi_{\text {split }} \mid C$ lifts to a $\mathbf{E}_{r}$-bundle, which is automatically topologically trivial since $\mathbf{E}_{r}$ is simply connected.

We have the exact sequence

$$
0=H^{1}(\widetilde{X} ; \operatorname{ad} \widetilde{\Xi}) \rightarrow H^{1}(C ; \operatorname{ad} \widetilde{\Xi} \mid C) \rightarrow H^{2}\left(\widetilde{X} ; \operatorname{ad} \widetilde{\Xi} \otimes \mathcal{O}_{\widetilde{X}}(-C)\right) .
$$

By Lemma 3.6, $H^{2}\left(\widetilde{X}\right.$; ad $\left.\widetilde{\Xi} \otimes \mathcal{O}_{\widetilde{X}}(-C)\right)=0$. Hence $H^{1}(C$; ad $\widetilde{\Xi} \mid C)=0$ as well, so that $\widetilde{\Xi} \mid C$ is rigid. But, since the trivial bundle is semistable, the only rigid $\widetilde{\mathbf{E}}_{r}$-bundle on $C$ is the trivial bundle. Thus $\widetilde{\Xi} \mid C$ is trivial.

Next we construct the bundle $\widetilde{\Xi}$ :

Lemma 3.8. There exists an $\widetilde{H} U$-bundle $\Xi_{\widetilde{H} U}$ lifting $\Xi_{\widetilde{H}}$ such that, for $\widetilde{\Xi}=\Xi_{\widetilde{H} U} \times_{\widetilde{H} U} \widetilde{\mathbf{E}}_{r}$, and for every $D \in\left|-K_{\tilde{X}}\right|$, we have $h^{0}(D ; \operatorname{ad} \widetilde{\Xi} \mid D)=r+1$.

Proof. By Lemma 3.5, it suffices to prove the result for one choice of $D \in\left|-K_{\tilde{X}}\right|$, which we may assume to be a smooth elliptic curve. By [3], there exists a $\widetilde{\mathbf{E}}_{r}$-bundle $\widetilde{\xi}$ of the form $\xi_{\widetilde{H} U} \times_{\widetilde{H} U} \widetilde{\mathbf{E}}_{r}$, such that (i) $h^{0}(D ;$ ad $\widetilde{\xi})=r+1$, and (ii) $\xi_{\widetilde{H} U}$ is a lift to $\widetilde{H} U$ of the bundle $\xi_{\widetilde{H}}=\Xi_{\widetilde{H}} \mid D$. Let us show that we can find an $\widetilde{H} U$-bundle $\Xi_{\widetilde{H} U}$ such that $\Xi_{\widetilde{H} U} \mid D=\xi_{\widetilde{H} U}$. 
To make the construction, choose any decreasing filtration $U=U_{0} \supseteq U_{1} \supseteq \cdots \supseteq U_{N} \supseteq$ $U_{N+1}=\{1\}$ of $U$ by normal $\widetilde{H}$-invariant subgroups such that, for every $i, U_{i} / U_{i+1}$ is contained in the center of $U / U_{i+1}$. It follows that $U_{i} / U_{i+1}$ is a vector group which is a direct sum of root spaces $\mathfrak{g}^{\alpha}, \alpha \in R_{i}$, say, and $\widetilde{H}$ acts on $U_{i} / U_{i+1}$ in the usual way. By induction on $i$, starting with $i=0$, it suffices to show the following: Given $i$, suppose that we have found an $\widetilde{H} U / U_{i}$-bundle $\Xi_{\widetilde{H} U / U_{i}}$ such that $\Xi_{\widetilde{H} U / U_{i}} \mid D \cong \xi_{\widetilde{H} U} / U_{i}$. Then we can lift $\Xi_{\widetilde{H} U / U_{i}}$ to a $\widetilde{H} U / U_{i+1}$-bundle $\Xi_{\widetilde{H} U / U_{i+1}}$ such that $\Xi_{\widetilde{H} U / U_{i+1}} \mid D \cong \xi_{\widetilde{H} U} / U_{i+1}$. By general formalism (we use the notation of [四, Appendix]), the obstruction to lifting $\Xi_{\widetilde{H} U / U_{i}}$ to some $\widetilde{H} U / U_{i+1}$-bundle lives in the cohomology group $H^{2}\left(\widetilde{X} ;\left(U_{i} / U_{i+1}\right)\left(\Xi_{\widetilde{H} U / U_{i}}\right)\right)$. But

$$
\left.\left(U_{i} / U_{i+1}\right)\left(\Xi_{\widetilde{H} U / U_{i}}\right)\right) \cong \bigoplus_{\alpha \in R_{i}} L_{\alpha}
$$

and $H^{2}\left(\widetilde{X} ; L_{\alpha}\right)=0$. Thus there is a lift of $\Xi_{\widetilde{H} U / U_{i}}$ to some $\widetilde{H} U / U_{i+1}$-bundle $\Xi_{\widetilde{H} U / U_{i+1}}$. Let $\xi_{\widetilde{H} U / U_{i+1}}=\Xi_{\widetilde{H} U / U_{i+1}} \mid D$. Then $\xi_{\widetilde{H} U / U_{i+1}}$ and $\xi_{\widetilde{H} U} / U_{i+1}$ are two lifts of $\xi_{\widetilde{H} U} / U_{i}$ to $\widetilde{H} U / U_{i+1}$, and as such they differ by the action of $H^{1}\left(D ;\left(U_{i} / U_{i+1}\right)\left(\xi_{\widetilde{H} U} / U_{i}\right)\right.$. On the other hand,

$$
\left(U_{i} / U_{i+1}\right)\left(\xi_{\widetilde{H} U} / U_{i}\right) \cong \bigoplus_{\alpha \in R_{i}}\left(L_{\alpha} \mid D\right) .
$$

By the last sentence of Lemma 3.3, since all of the roots in $R_{i}$ are positive, the restriction $\operatorname{map} H^{1}\left(\widetilde{X} ; L_{\alpha}\right) \rightarrow H^{1}\left(D ; L_{\alpha} \mid D\right)$ is an isomorphism for every $\alpha \in R_{i}$. Thus, we can adjust the lift $\Xi_{\widetilde{H} U / U_{i+1}}$ by the action of $H^{1}\left(\widetilde{X} ;\left(U_{i} / U_{i+1}\right)\left(\Xi_{\widetilde{H} U / U_{i}}\right)\right)$ so that $\Xi_{\widetilde{H} U / U_{i+1}} \mid D \cong$ $\xi_{\widetilde{H} U} / U_{i+1}$. This completes the inductive step.

Parts (i)-(iii) of Theorem 3.1 now follow from Lemma 3.8 and Lemma 3.5. Part (iv) follows from Lemma 3.7 and Lemma 3.4. Finally, we must check the uniqueness statement, that any bundle satisfying (i) and (ii) of Theorem 3.1 is isomorphic to $\widetilde{\Xi}$. Since $H^{2}\left(\widetilde{X} ; \operatorname{ad} \Xi_{\text {split }}\right)=0$, the deformations of $\Xi_{\text {split }}$ are unobstructed, and there exists a germ of a complex manifold, $\mathcal{U}$ which is the base space of a local semiuniversal deformation of $\Xi_{\text {split. }}$. By a standard argument (cf. [身, Lemma 4.1.1] for a related construction), given any extension of $\Xi_{\text {split }}$ to an $\widetilde{H} U$-bundle $\widehat{\Xi}$, there is a family of bundles over $\widetilde{X} \times \mathbb{C}$ which restrict to $\widehat{\Xi} \times{ }_{\widetilde{H} U} \widetilde{\mathbf{E}}_{r}$ over $\mathbb{C}-\{0\}$ and to $\Xi_{\text {split }}$ over 0 , and hence there are points of $\mathcal{U}$ corresponding to $\widehat{\Xi} \times \widetilde{H}_{U} \widetilde{\mathbf{E}}_{r}$. The subset $\mathcal{U}^{\prime}$ of $\mathcal{U}$ defined by bundles $\Upsilon$ such that $H^{1}(\widetilde{X} ; \operatorname{ad} \Upsilon)=0$ is the complement of a proper analytic subvariety and hence is open and dense and therefore connected. Since $\widetilde{\Xi}$ is rigid, the subset of $\mathcal{U}^{\prime}$ corresponding to bundles isomorphic to $\widetilde{\Xi}$ is open, as is its complement in $\mathcal{U}^{\prime}$. Hence every point of $\mathcal{U}^{\prime}$ corresponds to $\widetilde{\Xi}$, proving the uniqueness.

Remark 3.9. The proof actually shows the following. Let $G$ be the reductive subgroup of $\widetilde{\mathbf{E}}_{r}$ whose maximal torus is $\widetilde{H}$ and whose Lie algebra contains all of the root spaces corresponding to the effective roots. Then $\widetilde{\Xi}$ reduces to a Borel subgroup $\widetilde{H} U^{\prime}$ of $G$, where $U^{\prime}$ is the unipotent radical of the Borel, and $\Xi$ reduces to a $G$-bundle. 


\section{Restriction to hyperplane sections}

We now relate the restriction of this construction to smooth hyperplane sections to the correspondence outlined in [6, §2]. Let $X$ be a del Pezzo surface of degree $r$ with at worst rational double points and let $D$ be a smooth hyperplane section. Let $\varphi: D \rightarrow E$ be an isomorphism such that $\varphi^{*} \mathcal{O}_{E}\left((9-r) p_{0}\right)=\mathcal{O}_{X}(D) \mid D$. Then, following [6, §2], we define the period point of $(X, D, \varphi)$ in $\left(E \otimes_{\mathbb{Z}} \Lambda\right) / W$ as follows: Fix a marking $\psi$ and use it to identify $\widetilde{\Lambda}$ with Pic $\widetilde{X}$. Given $\alpha \in \Lambda$, the line bundle $\left(\varphi^{*}\right)^{-1}\left(L_{\alpha}\right)$ is a line bundle of degree zero on $E$, and hence defines a point in $\operatorname{Pic}^{0} E \cong E$. This defines a homomorphism $\Lambda \rightarrow E$. We can extend this to a homomorphism $\widetilde{\pi}: \widetilde{\Lambda} \rightarrow E$ in the following way. Given $\alpha \in \widetilde{\Lambda}$, let $k=\operatorname{deg}\left(L_{\alpha} \mid D\right)$. Define $\tilde{\pi}(\alpha)$ to be the point corresponding to the line bundle $\left(\varphi^{*}\right)^{-1}\left(L_{\alpha}\right) \otimes \mathcal{O}_{E}\left(-k p_{0}\right)$. Then $\widetilde{\pi}$ is a homomorphism, and $\widetilde{\pi}(\kappa)=0$. Hence there is an induced homomorphism $\widetilde{\Lambda} / \mathbb{Z} \cdot \kappa \rightarrow E$. Since the intersection pairing identifies $(\widetilde{\Lambda} / \mathbb{Z} \cdot \kappa)^{*}$ with $\Lambda$, we see that this homomorphism is equivalent to an element $\pi$ of $E \otimes_{\mathbb{Z}} \Lambda$. Changing the choice of the marking $\psi$ amounts to acting on $\Lambda$ via $W$, so that the period point is invariantly defined in $\left(E \otimes_{\mathbb{Z}} \Lambda\right) / W$.

Suppose that $\widetilde{\xi}$ is an $\widetilde{\mathbf{E}}_{r}$-bundle over $E$ and that $\lambda$ is a $\mathbb{C}^{*}$-bundle over $E$. The inclusion of $\mathbb{C}^{*}$ into the center of $\widetilde{\mathbf{E}}_{r}$ allows us to define a new bundle $\widetilde{\xi} \otimes \lambda$. Let $\operatorname{det} \widetilde{\xi}$ be the $\mathbb{C}^{*}$-bundle induced by the surjection $\widetilde{\mathbf{E}}_{r} \rightarrow \mathbb{C}^{*}$. Note that

$$
\operatorname{det}(\widetilde{\xi} \otimes \lambda)=\operatorname{det} \widetilde{\xi} \otimes \lambda^{9-r} .
$$

Theorem 4.1. Given a triple $(X, D, \varphi)$, let $\varphi^{*}(\Xi \mid D)=\widetilde{\xi}$. Then there is a natural choice of $a \mathbb{C}^{*}$-bundle $\lambda$ for which there is a canonical reduction of the structure group of the bundle $\widetilde{\xi} \otimes \lambda$ to an $\mathbf{E}_{r}$-bundle $\xi$ over E. Moreover:

(i) The bundle $\xi$ is regular, i.e. $h^{0}(E ; \operatorname{ad} \xi)=r$.

(ii) The period point of the triple $(X, D, \varphi)$ in $\left(E \otimes_{\mathbb{Z}} \Lambda\right) / W$ corresponds to the point defined by $\xi$, viewing $\left(E \otimes_{\mathbb{Z}} \Lambda\right) / W$ as the moduli space of semistable $\mathbf{E}_{r}$-bundles over $E$.

Proof. It is easy to check via the exact sequence

$$
\{1\} \rightarrow \mathbf{E}_{r} \rightarrow \widetilde{\mathbf{E}}_{r} \rightarrow \mathbb{C}^{*} \rightarrow\{1\}
$$

that the obstruction to lifting $\widetilde{\xi} \otimes \lambda$ to a $\mathbf{E}_{r}$-bundle vanishes if and only if the bundle $\operatorname{det}(\widetilde{\xi} \otimes \lambda)$ is trivial. Moreover, using the above sequence and the fact that there is a subgroup of $\operatorname{Aut}(\widetilde{\xi} \otimes \lambda)$ isomorphic to $\mathbb{C}^{*}$ which surjects onto $\operatorname{Aut}(\operatorname{det}(\widetilde{\xi} \otimes \lambda)) \cong \mathbb{C}^{*}$ any lift of $\widetilde{\xi} \otimes \lambda$ to $\mathbf{E}_{r}$ is unique up to isomorphism. Also, it is easy to verify that $\operatorname{det}(\widetilde{\xi} \otimes \lambda)=\operatorname{det}\left(\widetilde{\xi}_{\widetilde{H}} \otimes \lambda\right)$, where $\widetilde{\xi}_{\widetilde{H}}$ is the corresponding $\widetilde{H}$-bundle.

The exact sequence of tori

$$
\{1\} \rightarrow H \rightarrow \widetilde{H} \rightarrow \mathbb{C}^{*} \rightarrow\{1\}
$$


corresponds on the level of fundamental groups to

$$
0 \rightarrow \Lambda \rightarrow \widetilde{\Lambda} \rightarrow \mathbb{Z} \rightarrow 0
$$

where the surjection $\widetilde{\Lambda} \rightarrow \mathbb{Z}$ is given by $\lambda \mapsto\langle\lambda, \kappa\rangle$. It follows from Lemma 2.1 that $\operatorname{det} \Xi_{\text {split }}=\operatorname{det} \Xi_{\widetilde{H}}$ corresponds to the line bundle $\left(K_{\widetilde{X}}\right)^{-1}$. Thus, det $\widetilde{\xi}$ corresponds to the line bundle $\mathcal{O}_{E}\left((9-r) p_{0}\right)$. It follows that, if we set $\lambda=\mathcal{O}_{E}\left(-p_{0}\right)$, then $\operatorname{det}(\widetilde{\xi} \otimes \lambda)$ is trivial and thus $\widetilde{\xi} \otimes \lambda$ lifts to a $\mathbf{E}_{r}$-bundle $\xi$. The regularity of $\xi$ follows from the regularity of $\widetilde{\xi}$.

Finally, let us check that the period point of $(X, D, \varphi)$ corresponds to the point it defines in the moduli space. Since this point is independent of the S-equivalence class of $\xi$, we may as well work with $\varphi^{*}\left(\Xi_{\text {split }} \mid D\right) \otimes \mathcal{O}_{E}\left(-p_{0}\right)$. Working instead with the $\widetilde{H}$-bundle $\xi_{\widetilde{H}}$, note that a $\widetilde{H}$-bundle over $E$ is the same as an element of Pic $E \otimes \widetilde{\Lambda}$. The bundle $\xi_{\widetilde{H}}$ is characterized by the property that, for $\alpha \in \widetilde{\Lambda} \cong \widetilde{\Lambda}^{*}$, the $\mathbb{C}^{*}$-bundle arising from the homomorphism $\widetilde{\Lambda} \rightarrow \mathbb{Z}$ is $\left(\varphi^{-1}\right)^{*}\left(L_{\alpha} \mid D\right)$. From this, it is clear that the period point agrees with the moduli point.

\section{Fundamental weights and the geometry of del Pezzo sur- faces}

In this section, we assume that $4 \leq r \leq 8$, so that the group $\mathbf{E}_{r}$ is simple. Also, we shall assume tacitly that $X=\tilde{X}$, i.e. that we are working with a smooth del Pezzo surface, unless otherwise specified.

Fix the basis $h, e_{1}, \ldots, e_{r}$ for $\widetilde{\Lambda}$ and view elements of $\widetilde{\Lambda}$ as defining homomorphisms $\Lambda \rightarrow \mathbb{Z}$ via the inner product (which we continue to assume is given by $h^{2}=1, e_{i}^{2}=-1$ ). In this way, there is a homomorphism from $\widetilde{\Lambda}$ to the set of weights on $\Lambda$ and $\lambda \in \widetilde{\Lambda}$ is the trivial weight if and only if $\lambda$ is an integral multiple of $\kappa=3 h-\sum_{i} e_{i}$.

As before, we choose the simple coroots $\alpha_{1}=e_{1}-e_{2}, \ldots, \alpha_{r-1}=e_{r-1}-e_{r}, \alpha_{r}=$ $h-e_{1}-e_{2}-e_{3}$. By our conventions on the sign of intersection form, the root dual to a coroot $\alpha$ is confusingly identified with $-\alpha \in \widetilde{\Lambda}$ via the intersection form. Let $\left\{\varpi_{i}\right\}$ be the dual basis to $\left\{\alpha_{i}\right\}$. For future reference, we give the list of all the positive coroots and the highest root:

Lemma 5.1. The positive roots are:

- $e_{i}-e_{j}$ for $i<j$;

- $h-e_{i}-e_{j}-e_{k}$;

- $(r \geq 6) 2 h-\sum_{i \in I} e_{i}$, where $\# I=6$;

- $(r=8) 3 h-2 e_{i}-\sum_{j \neq i} e_{j}$.

The highest root $\widetilde{\alpha}$ is given as follows: For $r=4,5, \widetilde{\alpha}=h-e_{r-2}-e_{r-1}-e_{r}$, for $r=6,7, \widetilde{\alpha}=2 h-\sum_{i=1}^{6} e_{r+1-i}$, for $r=8, \widetilde{\alpha}=3 h-\sum_{j=1}^{7} e_{j}-2 e_{8}$. 
A routine calculation gives:

Lemma 5.2. The dual basis element $\varpi_{i}$ is the image of the following element $\widetilde{\varpi}_{i}$ of $\widetilde{\Lambda}$ :

- $\widetilde{\varpi}_{1}=h-e_{1}$, and the linear system of all divisors on $X$ whose cohomology class is $h-e_{1}$ is a pencil of conics on $X$;

- $\widetilde{\varpi}_{2}=2 h-e_{1}-e_{2}$ and the corresponding linear system is defined by a smooth rational curve of degree 4 .

- For $3 \leq i \leq r-1, \widetilde{\varpi}_{i}=e_{i+1}+\cdots+e_{r}$ corresponds to $r-i$ disjoint lines on $X$.

- $\widetilde{\varpi}_{r}=h$ and the corresponding linear system is defined by a twisted cubic.

The above lemma shows that, for example, a lift of the fundamental weight $\varpi_{1}$ may be identified with a particular pencil of conics on $X$. Of course, $W$ acts on the set of all weights. Via the marking, $W$ also acts on $H^{2}(X ; \mathbb{Z})$ and hence on the group of divisor classes of $X$. In fact, $W$ acts simply transitively on the set of blowdowns $X \rightarrow \mathbb{P}^{2}$ together with a labeling of the exceptional curves. We next show that the Weyl orbits of the corresponding fundamental weights may be identified with the corresponding geometric objects on $X$ :

Lemma 5.3. The Weyl group $W$ operates transitively on any one of the following sets of objects: disjoint sets of $k$ lines, $k \leq r-3$; pencils of conics; twisted cubics; linear systems of smooth rational curves of degree four.

Proof. Every set of $k \leq r-3$ disjoint lines can be completed to a set of $r$ disjoint lines. On the other hand, such a set defines a blowdown to $\mathbb{P}^{2}$ and hence a diagonal basis $\gamma, \epsilon_{1}, \ldots, \epsilon_{r}$ with $\gamma^{2}=1, \epsilon_{i}^{2}=-1$ and $\kappa=3 \gamma-\sum_{i} \epsilon_{i}$. The Weyl group acts transitively on such bases, by Lemma 1.1, and hence on the set of all sets of $k$ disjoint lines. A similar argument handles the case of $r-1$ disjoint lines such that the blowdown is $\mathbb{P}^{1} \times \mathbb{P}^{1}$.

Now suppose that $C$ is a twisted cubic on $X$, i.e. a smooth rational curve $C$ with $C^{2}=1$. The linear system $|C|$ defines a birational morphism to $\mathbb{P}^{2}$, and we have seen that all such are conjugate under the Weyl group. If $C$ is a conic, then it is easy to check that there is a blowdown to $\mathbb{P}^{2}$ such that $C=\gamma-\epsilon_{1}$ for $\gamma$ the pullback of a hyperplane class and $\epsilon_{1}$ an exceptional curve, and again all such are conjugate under $W$. Finally, if $C$ is a smooth rational quartic, then $|C|$ defines a birational morphism from $X$ to a quadric in $\mathbb{P}^{3}$, and since $X$ has no-2-curves, the quadric is smooth. We conclude in this case by the last sentence of the preceding paragraph.

Each $\varpi_{i}$ is the highest weight of an irreducible representation of $\mathbf{E}_{r}$, called a fundamental representation. We make some comments on the fundamental representations.

Minuscule representations. For $r=4$, every fundamental representation is minuscule. For $r=5$, the three minuscule representations are the standard representation, with highest weight $\varpi_{1}$, and the two half-spin representations, with highest weights $\varpi_{4}, \varpi_{5}$ respectively. For $r=6$, the two minuscule representations are the two representations with highest 
weights $\varpi_{1}, \varpi_{5}$. For $r=7$, the representation with highest weight $\varpi_{6}$ is the unique minuscule representation. For $r=8$, there are no minuscule representations.

The adjoint representation. This is the unique quasi-minuscule, non-minuscule representation. In all cases the highest weight is the class of $\kappa-\widetilde{\alpha}$ (recall our conventions on the signs of roots). For $r=4$, the adjoint representation is not fundamental. For $r=5,6,7,8$, it is respectively the representation with highest weight $\varpi_{2}, \varpi_{6}, \varpi_{1}, \varpi_{7}$.

Dual representations. Two irreducible representations are dual if the negative of the lowest weight of one is equal to the highest weight of the other. In particular, a representation is isomorphic to its dual if and only if the lowest weight is the negative of the highest weight $\mu$, or equivalently in terms of Weyl groups, if $-w_{0}(\mu)=\mu$, where $w_{0} \in W$ is the unique element sending positive roots to negative roots. For $\mu=\varpi_{\alpha}, \alpha$ a simple root, this is equivalent to: $\alpha$ is fixed by the outer automorphism $-w_{0}$ of the root system. For $E_{7}$ and $E_{8}$, every outer automorphism is trivial and this condition is automatic. In terms of the lattice $\widetilde{\Lambda}$, suppose that we are given lifts of $\varpi_{i}$ and $\varpi_{j}$ to elements $\widetilde{\varpi}_{i}$ and $\widetilde{\varpi}_{j}$ in $\widetilde{\Lambda}$. Then the representations corresponding to $\varpi_{i}$ and $\varpi_{j}$ are dual if and only if the sum of $\widetilde{\varpi}_{i}$ and a lift to $\widetilde{\Lambda}$ of the lowest weight corresponding to $\varpi_{j}$ add up to a multiple of $\kappa$. Clearly, this condition holds if and only if there is some $w \in W$ such that $\widetilde{\varpi}_{i}+w\left(\widetilde{\varpi}_{j}\right)=n \kappa$. For example, in case $r=6$, i.e. degree three, we can write the hyperplane section as the sum of a line and a conic. For $A_{4}$, the dualities amount to saying that we can write the hyperplane section of a degree 5 del Pezzo as the sum of a conic and a twisted cubic, or as a line plus a smooth rational curve of degree four. For $D_{5}$, we can write the hyperplane section of a degree 4 del Pezzo as the sum of a line and a twisted cubic, as the sum of two conics, or as four lines, grouped in two pairs of disjoint lines, and we can write twice the hyperplane section as a sum of two smooth rational curves of degree four. Similar but more complicated results hold for the smaller degrees.

The cubic form. Suppose that we are in the case $r=6$, i.e. the case of cubic surfaces. The weights of the 27-dimensional representation correspond to lines on the cubic surface. As was already known to Cartan [1, pp. 272-273], there is a symmetric cubic form on the 27-dimensional representation of $\mathbf{E}_{6}$, and in fact $\mathbf{E}_{6}$ is the group of automorphisms of this form. In terms of the geometry of the cubic surface, this form is determined by the following condition: it is nontrivial on the tensor product of three weight spaces if and only if the three corresponding lines sum up to a hyperplane section. It is an exercise that the Weyl group acts transitively on the set of all sets of three coplanar lines, so in fact up to a scalar multiple this is the only possible invariant cubic form.

To give a partial answer to the question of Manin posed in the introduction, the meaning of number of double sixes is that it is one-half the number of roots, which we can see as follows. A double six consists of two disjoint collections of lines $\left\{L_{1}, \ldots, L_{6}\right\}$ and $\left\{L_{1}^{\prime}, \ldots, L_{6}^{\prime}\right\}$ such that $L_{i} \cdot L_{j}=L_{i}^{\prime} \cdot L_{j}^{\prime}=0$ for all $i, j$, and $L_{i} \cdot L_{j}^{\prime}=\delta_{i j}$. Moreover, the six lines $\left\{L_{1}^{\prime}, \ldots, L_{6}^{\prime}\right\}$ are determined by the set $\left\{L_{1}, \ldots, L_{6}\right\}$. Instead of choosing a double six, choose one of the two collections of disjoint lines that it determines - there are 72 of these. Each such determines a blowdown to $\mathbb{P}^{2}$ and hence a root $2 \gamma-\epsilon_{1}-\cdots-\epsilon_{6}$, where 
the $\epsilon_{i}$ are the exceptional curves of the blowdown and $\gamma$ is the pullback of the hyperplane section. For example, for the standard blowdown we get the root $2 h-\sum_{i} e_{i}$. Since all roots are Weyl conjugate, we must get them all this way. The remaining question, concerning the 45 coplanar triples of lines, is connected, perhaps less explicitly, to the cubic form on the standard representation of $\mathbf{E}_{6}$.

We now consider the vector bundle induced from $\Xi$ under a representation of $\widetilde{\mathbf{E}}_{r}$. Because we assume that $X$ is smooth, $\Xi=\Xi_{\text {split }}$. Let $\rho: \mathbf{E}_{r} \rightarrow G L(N)$ be an irreducible representation, and suppose that we have extended $\rho$ to a representation $\widetilde{\rho}: \widetilde{\mathbf{E}}_{r} \rightarrow G L(N)$. Such an extension is equivalent to choosing an integer $b$ such that

$$
\rho(c)=\exp (2 \pi \sqrt{-1} b / d) \cdot \mathrm{Id}
$$

where $d=9-r$ is the order of $c$ (cf. (i) of Lemma 1.2). For example, there is a unique choice of $b$ with $0 \leq b \leq d-1$. The map

$$
(g, t) \in \mathbf{E}_{r} \times \mathbb{C}^{*} \mapsto \rho(g) \cdot t^{b} \mathrm{Id}
$$

then gives an extension $\widetilde{\rho}$. It is clear from the example at the end of Section 1 that, if $\rho$ is the irreducible with highest weight $\varpi_{r-1}$ and $c$ is the corresponding central element, then $\rho(c)=\exp (2 \pi \sqrt{-1} / d) \cdot$ Id and in fact $\widetilde{\mathbf{E}}_{r}$ is just $\rho\left(\mathbf{E}_{r}\right) \cdot \mathbb{C}^{*} \subseteq G L(N)$. In this case, we can always choose the extension $\widetilde{\rho}$ to be the inclusion. More generally, using (ii) of Lemma 1.2, an extension of $\rho$ to $\widetilde{\rho}$ corresponds to choosing a $\lambda \in \widetilde{\Lambda}$ such that the highest weight of $\rho$ is the image of $\lambda$ under the map from $\widetilde{\Lambda}$ to $\Lambda^{*}$ given by the inner product, and any two such differ by a multiple of $\kappa$.

For a weight $\lambda$ of $\widetilde{\rho}$, which we view via the intersection form as an element of $\widetilde{\Lambda}$, the corresponding line bundle is the line bundle we have denoted by $L_{\lambda}$. We have:

Proposition 5.4. Suppose that $X$ is smooth. Let $\widetilde{\rho}: \widetilde{\mathbf{E}}_{r} \rightarrow G L(N)$ be the representation defined by the lift $e_{r}$ of $\varpi_{r-1}$. Then, for $r \neq 8$, the induced vector bundle $\Xi \times_{\widetilde{\mathbf{E}}_{r}} \mathbb{C}^{N}$ is $\bigoplus_{i} \mathcal{O}_{X}\left(L_{i}\right)$, where the $L_{i}$ are the distinct lines on $X$. For $r=8, \Xi \times_{\widetilde{\mathbf{E}}_{8}} \mathbb{C}^{248} \cong \bigoplus_{i} \mathcal{O}_{X}\left(L_{i}\right) \oplus$ $\left(K_{X}^{-1}\right)^{\oplus 8}$.

Proof. For $r \neq 8$, this is clear by the remarks before the statement. For $r=8$, the adjoint bundle ad $\Xi \cong \bigoplus_{\alpha \in R} L_{\alpha} \oplus \mathcal{O}_{X}^{8}$, where $R \subseteq H^{2}(X ; \mathbb{Z})$ is the set of roots. The result then follows since $\Xi \times_{\widetilde{\mathbf{E}}_{8}} \mathbb{C}^{248} \cong \operatorname{ad} \Xi \otimes K_{X}^{-1}$.

For $r=6$, there is also the natural cubic form on the rank 27 vector bundle, with values in the line bundle $\mathcal{O}_{X}(D)=K_{X}^{-1}$, where $D$ is a hyperplane section of $X$, defined by the obvious map

$$
\mathcal{O}_{X}\left(L_{i}\right) \otimes \mathcal{O}_{X}\left(L_{j}\right) \otimes \mathcal{O}_{X}\left(L_{k}\right) \rightarrow \mathcal{O}_{X}\left(L_{i}+L_{j}+L_{k}\right)
$$

Finally, we discuss the structure of the bundle in case $X$ has rational double points. For simplicity, let us just consider the case where $r=6$ and there is exactly one double point whose preimage on $\widetilde{X}$ is $C$. For example, we could take $[C]=e_{1}-e_{2}$. The 27 classes 
$e_{i}, h-e_{i}-e_{j}, 2 h-\sum_{i \neq k} e_{i}$ which define lines on a smooth cubic - call these numerical lines - specialize to 6 pairs of the form $\{\lambda, \lambda+C\}$ corresponding to 6 lines on the singular cubic $X$ which pass through the double point, together with 15 lines which do not meet the double point. Let $L_{i}^{\prime}, i=1, \ldots, 6$ be the line bundles corresponding to numerical lines $\lambda$ such that $\lambda+C$ is also a numerical line. This is equivalent to: $L_{i}^{\prime} \cdot C=1$. Let $L_{j}^{\prime \prime}$, $j=1, \ldots, 15$ be the remaining classes, so that $L_{j}^{\prime \prime} \cdot C=0$. Finally, let $V_{2}$ be the unique rank two vector bundle on $\widetilde{X}$ which is a nontrivial extension

$$
0 \rightarrow \mathcal{O}_{\widetilde{X}}(C) \rightarrow V_{2} \rightarrow \mathcal{O}_{\widetilde{X}} \rightarrow 0
$$

It is easy to check that the map $H^{1}\left(\widetilde{X} ; \mathcal{O}_{\widetilde{X}}(C)\right) \rightarrow H^{1}\left(C ; \mathcal{O}_{\tilde{X}}(C) \mid C\right)=H^{1}\left(C ; \mathcal{O}_{C}(-2)\right)$ is an isomorphism. Thus, the extension $V_{2}$ restricts on $C$ to the nontrivial extension

$$
0 \rightarrow \mathcal{O}_{C}(-2) \rightarrow V_{2} \mid C \rightarrow \mathcal{O}_{C} \rightarrow 0
$$

It follows that $V_{2} \mid C \cong \mathcal{O}_{C}(-1) \oplus \mathcal{O}_{C}(-1)$. Thus, if we consider the rank 27 bundle

$$
\mathcal{V}=\bigoplus_{i=1}^{6}\left(V_{2} \otimes L_{i}^{\prime}\right) \oplus \bigoplus_{j=1}^{15} L_{j}^{\prime \prime},
$$

then $\mathcal{V}$ restricts to the trivial rank 27 bundle over $C$ and thus is pulled back from $X$. It is easy to see that $\mathcal{V}$ is the bundle induced from the standard representation of $\widetilde{\mathbf{E}}_{6}$. Similar but considerably more elaborate descriptions exist for the case of arbitrary rational double points.

\section{References}

[1] É. Cartan, Sur la structure des groupes de transformations finis et continus, Thèse (1894), in Euvres Complètes, Paris, Gauthier-Villars, 1952.

[2] M. Demazure, H. Pinkham and B. Teissier (eds.), Séminaire sur les Singularités des Surfaces, Lecture Note in Mathematics 777, Berlin Heidelberg New York, SpringerVerlag, 1980.

[3] R. Friedman and J.W. Morgan, Holomorphic principal bundles over elliptic curves, math.AG/9811130.

[4] R. Friedman and J.W. Morgan, Holomorphic principal bundles over elliptic curves II: The parabolic construction, math.AG/0006174.

[5] R. Friedman and J.W. Morgan, Holomorphic principal bundles over elliptic curves III: Singular curves and fibrations, in preparation.

[6] R. Friedman, J.W. Morgan, and E. Witten, Principal G-bundles over elliptic curves, Math. Research Letters 5 (1998), 97-118. 
[7] N.C. Leung, ADE-bundle over rational surfaces, configuration of lines and rulings, math.AG/0009192.

[8] Y. Manin, Cubic Surfaces: Algebra, Geometry, Arithmetic, Second edition, Translated from the Russian by M. Hazewinkel. Amsterdam-New York, North-Holland, 1986.

[9] M. Reid, Nonnormal del Pezzo surfaces, Publ. RIMS 30 (1994), 695-727.

Department of Mathematics

Columbia University

New York, NY 10027

USA

rf@math.columbia.edu, jm@math.columbia.edu 